\title{
Increasing Lifetimes and the Growing Saddles of Shear Flow Turbulence
}

\author{
Tobias Kreilos, ${ }^{1,2, *}$ Bruno Eckhardt, ${ }^{2,3}$ and Tobias M. Schneider ${ }^{1,4, \dagger}$ \\ ${ }^{1}$ Max Planck Institute for Dynamics and Self-Organization, Am Fassberg 17, 37077 Göttingen, Germany \\ ${ }^{2}$ Fachbereich Physik, Philipps-Universität Marburg, 35032 Marburg, Germany \\ ${ }^{3}$ J.M. Burgerscentrum, Delft University of Technology, Mekelweg 2, 2628 CD Delft, The Netherlands \\ ${ }^{4}$ Institute of Mechanical Engineering, École Polytechnique Fédérale de Lausanne, 1015 Lausanne, Switzerland
}

(Received 24 July 2013; published 31 January 2014)

\begin{abstract}
In linearly stable shear flows, turbulence spontaneously decays with a characteristic lifetime that varies with Reynolds number. The lifetime sharply increases with Reynolds number so that a possible divergence marking the transition to sustained turbulence at a critical point has been discussed. We present a mechanism by which the lifetimes increase: in the system's state space, turbulent motion is supported by a chaotic saddle. Inside this saddle a locally attracting periodic orbit is created and undergoes a traditional bifurcation sequence generating chaos. The formed new "turbulent bubble" is initially an attractor supporting persistent chaotic dynamics. Soon after its creation, it collides with its own boundary, by which it becomes leaky and dynamically connected with the surrounding structures. The complexity of the chaotic saddle that supports transient turbulence hence increases by incorporating the remnant of a new bubble. As a result, the time it takes for a trajectory to leave the saddle and decay to the laminar state is increased. We demonstrate this phenomenon in plane Couette flow and show that characteristic lifetimes vary nonsmoothly and nonmonotonically with Reynolds number.
\end{abstract}

DOI: 10.1103/PhysRevLett.112.044503

PACS numbers: 47.27.Cn, 47.20.Ft, 47.52.+j

The emergence of turbulence in shear flows such as pipe or Couette flow has puzzled scientists for more than a century, because the transition is not associated with a linear instability of the laminar base flow [1] and requires perturbations of finite amplitude [2,3]. Once triggered, turbulence in these flows is not persistent but can spontaneously decay [4]. This phenomenology can be understood in the context of dynamical systems, where the structure of the state space - the space of all instantaneous velocity fields-is investigated. The state space is filled with a multitude of unstable invariant solutions and a turbulent trajectory transiently visits those solutions before it eventually escapes their neighborhood and decays to the laminar state. This picture of a chaotic saddle that supports transient turbulence has been corroborated by the direct identification of numerous invariant solutions and some dynamical connections between them both in plane Couette and pipe flow [5-7]. Further support comes from the distribution of transient lifetimes, which is found to be an exponential and thus of the form expected for chaotic saddles. The characteristic time of the exponential distribution increases sufficiently rapidly with Reynolds number Re to explain why decays are rarely observed at high Re. How exactly the characteristic lifetime $\tau$ increases has attracted much recent attention, specifically the question of whether there is a critical Re at which lifetimes diverge and turbulence becomes sustained [4, 8-22].

While previous studies have characterized a functional form of the dependence of $\tau$ on Re, we here present a mechanism that leads to increasing lifetimes. Within the picture of a turbulent trajectory meandering between invariant solutions before eventually escaping the chaotic saddle and decaying to laminar flow, increasing turbulent lifetimes are directly linked to changes of the chaotic saddle structure. We describe a sequence of events within a small range of Re that add an infinite number of invariant solutions to the chaotic saddle. Thereby the complexity of the chaotic saddle grows and lifetimes increase. During the process the lifetimes diverge for some Reynolds numbers, leading to a nonmonotonic and nonsmooth variation of $\tau$ with Re.

In extended flow systems, the local relaminarization of turbulence can be overcome by spatial spreading so that turbulence may become sustained in a percolation-like phase transition despite its local decay $[23,24]$. To separate the strongly increasing variations of $\tau$ in a confined domain from the spatial spreading of turbulence, we consider plane Couette flow in a small periodic box. The box is chosen such that the flow is described by a comparably low number of degrees of freedom $\left(\mathcal{O}\left(10^{5}\right)\right)$. This allows for a detailed analysis of the high-dimensional state space, which remains computationally challenging; specifically, we consider the system studied previously in Kreilos and Eckhardt [6], which is a bit longer and narrower than the minimal flow unit studied for example in [25]. Plane Couette flow is the flow of a fluid between two parallel plates moving at constant speed in opposite direction governed by the incompressible Navier Stokes equations $\partial_{t} \mathbf{u}+(\mathbf{u} \cdot \nabla) \mathbf{u}=$ $-\nabla p+\mathrm{Re}^{-1} \Delta \mathbf{u}$ together with the continuity equation $\nabla \cdot \mathbf{u}=0$ and boundary conditions $\mathbf{u}= \pm \mathbf{e}_{x}$ at $y \pm 1$, where $p$ is the pressure and $y$ is the wall-normal coordinate. 
The Reynolds number is defined by $\operatorname{Re}=U_{0} h / \nu$ with $U_{0}$ half the relative plate velocity, $h$ half the plate separation and $\nu$ the viscosity of the fluid and using $U_{0}$ and $h$ as velocity and length scale, respectively. Direct numerical simulations are performed using the pseudospectral solver channel flow [20,26] developed by John F. Gibson. The computational domain is of size $L_{x} \times 2 h \times$ $L_{z}=2 \pi \times 2 \times \pi$ with a resolution of $48 \times 33 \times 48$ modes subject to periodic boundary conditions in the homogeneous directions and enforced shift-and-reflect symmetry $(u, v, w)[x, y, z] \equiv \sigma_{S R}(u, v, w)[x, y, z]=(u, v,-w) \times$ $\left[x+L_{x} / 2, y,-z\right]$.

Plane Couette flow is linearly stable for all Re and the first (known) nontrivial invariant solutions are the NagataBusse-Clever (NBC) states [25,27,28], a pair of fixed points that are created in a saddle-node bifurcation. In our domain the bifurcation takes place at $R e=163.8$ and the upperbranch solution (with higher energy) is attracting while the lower branch has a single unstable direction. The lower branch's stable manifold forms the separating boundary between trajectories approaching the upper branch and the laminar state [29,30]. Increasing Re, the upper branch undergoes a period doubling cascade, generating chaos. At $\mathrm{R} e=$ 188.51 the chaotic attractor formed around the upper branch subsequently collides with the lower branch. In this boundary crisis [6], the chaotic attractor becomes "leaky" and turns into a chaotic saddle, from which trajectories eventually escape. Similar scenarios have been found for periodic [31] and localized [32] structures in pipe flow as well as in magnetohydrodynamics [33], indicating a generic mechanism by which the saddle that supports first short-lived turbulent transients in shear flows is created. Similar scenarios are well-known in low-dimensional dynamical systems [34-36].

As Re increases, the chaotic saddle grows to fill a larger part of state space. In Fig. 1 this is visualized for three successively increasing Re by plotting the lifetimes of initial conditions, see figure caption for details. Blue regions correspond to initial conditions that directly laminarize while the chaotic saddle appears as colorful fractal regions of rapidly varying lifetimes indicative of the sensitive dependence on initial condition. Since the lower branch solution has a single unstable direction, its stable manifold is of co-dimension one and the general topology of state space can be understood in a two-dimensional section. For $\operatorname{Re}=210$ (panel a), the saddle only occupies a small area. As Re increases (panels b and c), the colorful fractal regions marking the saddle both grow and the variations inside become denser. As expected, the survival probabilities, shown in panel d, show exponential tails indicating a constant escape probability from the saddle. Their slope, the characteristic lifetime $\tau$, increases with Re as the saddle structure fills the domain.

How does the complexity of the saddle increase? We explore one mechanism and specifically demonstrate a sequence of bifurcation events, in which an infinite number
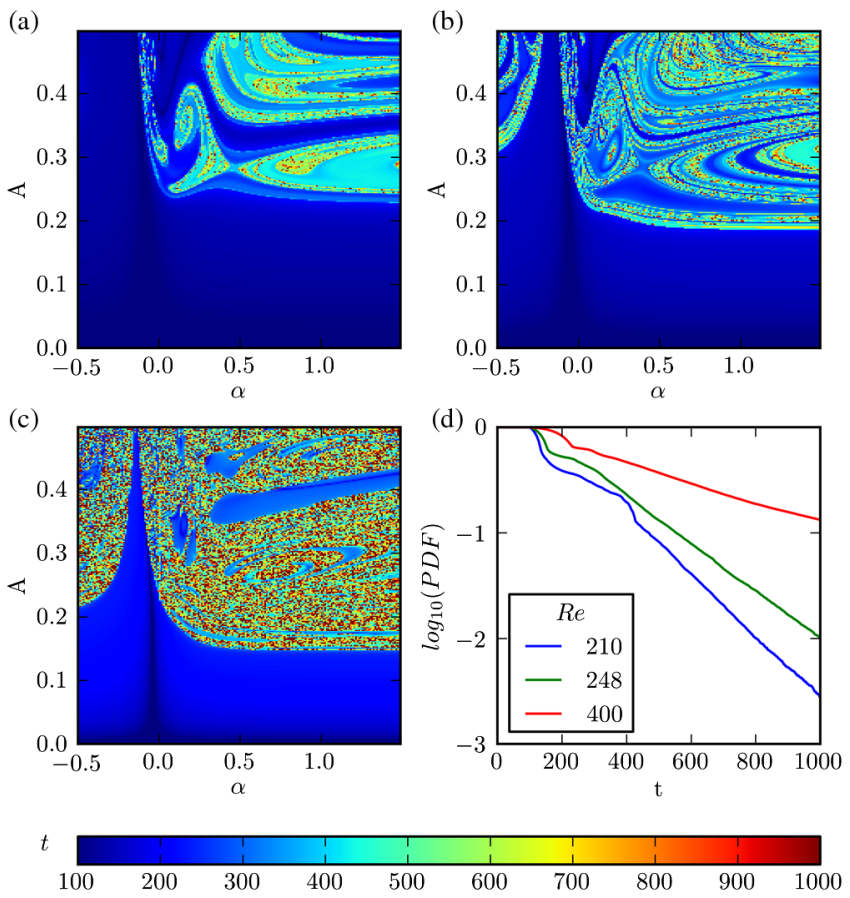

FIG. 1 (color online). (a)-(c): The growing turbulent saddle in state space for different Reynolds numbers, visualized by the lifetime of initial conditions in a 2D section. The 50,000 initial conditions in each panel are chosen by interpolating between lower- and upper-branch NBC solutions on the $x$ axis and rescaling the perturbation amplitude $A$ on the $y$ axis. The $x$ axis is rescaled such that the $L_{2}$ distance of the NBC states is proportional to their distance in the projection, giving a physical meaning to the scales in the figure, $u=A u_{\alpha} /\left\|u_{\alpha}\right\| u_{\alpha}=u_{L B}+\alpha\left(u_{U B}-u_{L B}\right) /$ $\sqrt{\left\|u_{U B}-u_{L B}\right\|^{2}-\left(\left\|u_{U B}\right\|-\left\|u_{L B}\right\|\right)^{2}}$. In the sequence, (a) $\mathrm{Re}=210$, (b) $\mathrm{Re}=248$, (c) $\mathrm{Re}=400$, the saddle grows while it develops ever finer striations, indicating that the structures supporting the saddle get denser. (d) Survival probabilities for the presented Reynolds numbers show clear exponential tails with increasing slope, indicating a characteristic lifetime that increases with Re.

of invariant solutions is added to the saddle, leading to an increase in lifetimes; the mechanism bears a strong resemblance with the events at the creation of the saddle as summarized above. At $\mathrm{Re}=249.01$ a local saddle-node bifurcation creates a pair of new solutions which are not fixed points as the NBC states but periodic orbits of period $T \simeq 60$. Of those, as for the NBC states, the lower branch has a single unstable direction and the upper branch solution is an attractor. The stable periodic orbit is surrounded by its basin of attraction, visible in Fig. 2(b) at $R e=249.1$. As Re increases, the orbit undergoes a bifurcation cascade, creating infinitely many invariant states and generating a local chaotic attractor (panel c). On further increasing $\mathrm{Re}$, the chaotic attractor grows until at $\operatorname{Re}_{c}=250.13$ it collides with its own basin boundary in a crisis bifurcation. While the signature of the basin of attraction of the former attractor is still visible for $\operatorname{Re}>\operatorname{Re}_{c}$ (panel d), trajectories 

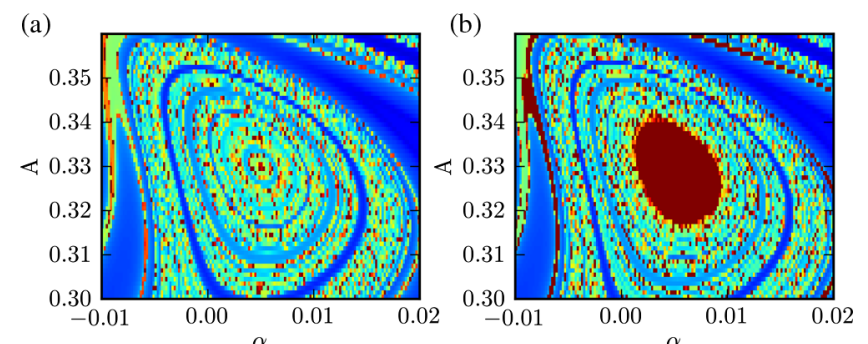

(c)
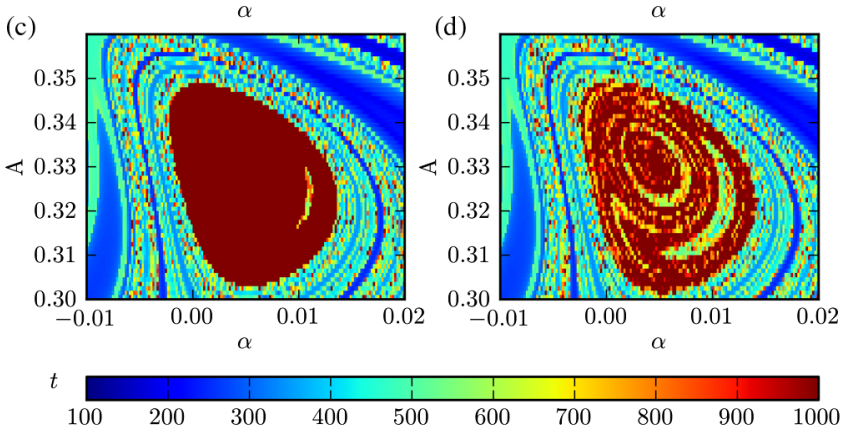

FIG. 2 (color online). Growth of the turbulent saddle via the emergence of a chaotic attractor followed by its destruction in a boundary crisis. Visualized is a small region of the saddle in a projection similar to Fig. 1 but spanned by the new pair of periodic orbits at $\mathrm{Re}=250$ (with the phase such that energy is maximal). Within the pre-existing chaotic saddle at $R e=248.5$ (a) the saddle-node bifurcation creates the new pair of two periodic orbits, of which one is stable. Its basin of attraction at $\mathrm{Re}=249.1$ (b) just after the bifurcation is visible as the red region of initial conditions that never reach the laminar state. On further increasing $\mathrm{Re}$, the orbit undergoes a bifurcation cascade generating a chaotic attractor, shown at $\operatorname{Re}=250.1$ (c), whose basin of attraction has grown. At $\mathrm{Re}_{c}=250.13$ the attractor collides with its boundary in a crisis bifurcation. Beyond the crisis bifurcation, the attractor has turned into a repellor and it is now a part of the turbulent saddle. The remnant of the attractor's basin of attraction remains visible at $\operatorname{Re}=250.25$ (d), where now the new "inner" saddle is immersed in the "outer" one.

may now escape from the newly created "inner" saddle into the already existing "outer" one. Thus at the crisis bifurcation the infinitely many invariant solutions from the former chaotic attractor are added to the turbulent saddle. As before, the two-dimensional visualization captures the essential features of the process since the lower-branch orbit has only a single unstable direction and hence a stable manifold of co-dimension one.

Over the sequence of these bifurcations, the decay rate from the turbulent saddle to the laminar state varies nonmonotonically. Before the saddle-node bifurcation at $\operatorname{Re}=249.01$, the survival probabilities are distributed exponentially [Fig. 3(a)]. Beyond $\operatorname{Re}=249.01$, there are initial conditions that are trapped inside the stable region and never decay. Thus, the overall distribution is a linear superposition of an exponential and a constant. The contribution of the nondecaying trajectories increases with higher Reynolds numbers as the attractor grows to fill more of the projection plane. After the boundary crisis at $\mathrm{Re}=250.13$,

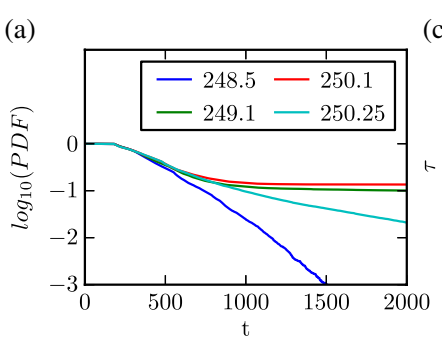

(c)

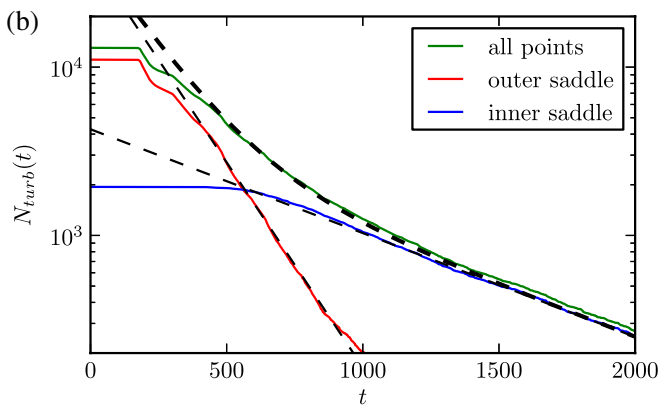

FIG. 3 (color online). Variations of lifetimes with Reynolds number. (a) Survival probability calculated from the data in Fig. 2 for all four Re. Before the saddle-node bifurcation $(\operatorname{Re}=248.5)$, survival probabilities are exponentially distributed. After the bifurcation, the attractor causes an offset corresponding to the initial conditions that never decay. After the boundary crisis $(\operatorname{Re}=250.25)$, the distribution is nonexponential. (b) Detailed analysis of the lifetime statistics at $\mathrm{Re}=250.25$, with the total number of trajectories that have not decayed up to a given time $t$ as a function of time. In Fig. 2(d) the shape of the former attractor is still visible and allows to calculate the characteristic lifetime separately for initial conditions from the new, inner saddle and the outer saddle. Both show exponential tails with a characteristic lifetime of 700 and 180, respectively. The thick dashed line on top shows the weighted average of the two exponentialsit very well fits the actually observed distribution (green), apart from the trajectories that decay immediately. (c) Reynolds number vs. characteristic lifetime $\tau$ as a function of Re, calculated separately for initial conditions from the inner and outer saddle. Lifetimes for the outer saddle are almost constant. The lifetime corresponding to the inner saddle diverges up to the crisis bifurcation, after which, it drops drastically with a power-law behavior and levels slightly above the value of the outer saddle, showing that lifetimes have increased.

the new bubble becomes a part of the turbulent saddle. Initially the escape rate from the newly created embedded part of the saddle differs substantially from the outer saddle's escape rate. Thus, for short observation times the distribution is a linear superposition of two different exponentials resulting in a nonexponential variation.

To quantify the variation of lifetimes due to both parts of the saddle, we separate initial conditions into two groups corresponding to the location of the former attractor in the discussed projection plane and compute lifetimes for both parts of the saddle independently. This is detailed for $\mathrm{Re}=250.25$ in Fig. 3(c), where instead of a normalized survival probability we plot the absolute number of initial 
conditions that survive for a given time. Since the initial conditions are uniformly distributed over the area shown in Fig. 2 the absolute numbers are a measure of the state space area that contributes to a given lifetime. The total number of data points is separated into those initial conditions from the inner and outer saddle. In Fig. 3 both individual distributions show clear exponential tails with characteristic lifetimes of 700 and 180, respectively. The sum of the two exponentials, weighted by the number of initial conditions in the two regions, (thick black line) almost perfectly recovers the total distribution. Similar nonexponential distributions due to several weakly connected saddle structures have also been studied in Hamiltonian systems, where nested saddles are shown to generate algebraic lifetime distributions [37].

The variation of $\tau$ with $\operatorname{Re}$ for both the inner and outer saddle region is shown in Fig. 3(b). While it remains almost constant for the outer region, in the inner region the lifetime diverges while the attractor exists and drops after the boundary crisis, well approximated by a power law with exponent -2.1 . The characteristic lifetime levels off slightly above the value of the outer saddle, indicating that trajectories that leave the inner saddle have to first pass through the outer one before decaying. Consequently, those trajectories have longer lifetimes. At higher Re, additional heteroclinic bifurcations will enhance the dynamical connectivity between the inner and outer saddle region which finally translates the higher lifetimes from the inner region to the whole saddle. Thereby, the characteristic lifetime of the saddle increases when it expands due to the incorporation of the newly created turbulent bubble.

We have found sudden expansions of the structure of the chaotic saddle at discrete Reynolds numbers as discussed for $R e \simeq 250$ at various $R e$, indicating that the mechanism is a generic way to increase the complexity of the state space. While the presented example of a stable periodic orbit emerging in a saddle-node bifurcation followed by a chaotic cascade and a boundary crisis of a transiently existing attractor is observed for many parameters in our system, a more general variant of the discussed mechanism starts with unstable states. The sequence of events described above then appears in a weakly unstable subspace of the full system. This does not involve a global attractor for intermediate $\mathrm{Re}$ and is hence more difficult to detect as the lifetimes do not diverge. Yet, the same scenario still increases the complexity of the saddle, leading to growing lifetimes.

The formation of turbulent bubbles and their destruction at discrete $\mathrm{Re}$ values constitutes abrupt changes in the state space of shear flows. The described mechanism naturally leads to nonsmooth and nonmonotonic variations of $\tau$ as well as nonexponential lifetime statistics. While we have found these features for intermediate Re in the considered system, they have not been reported yet for experimental lifetime studies. Apart from the symmetry restriction, which is only possible in numerics, one challenge is that the bifurcations become dense in $\mathrm{Re}$ and begin to overlap. To resolve this, very fine steps in Re are needed, as well as a very exact control of the applied perturbation $[38,39]$. In a sufficiently coarse grained analysis, an apparent smooth variation emerges due to the collective effect of many bifurcations.

The mechanism we explored in this Letter allows us to directly relate state-space structures and their variation to statistical properties of transitional flows. While our analysis is focused on plane Couette flow, recent investigations of pipe flow and even mangnetohydrodynamic systems suggest that the described mechanism by which turbulent saddles grow in complexity are very generic. This emphasizes the significance of low-dimensional dynamical systems theory for understanding the physics of fluid mechanical systems with a subcritical transition to turbulence.

The authors acknowledge financial support by the Niedersächsisches Ministerium für Wissenschaft und Kultur and the Deutsche Forschungsgemeinschaft. We thank Hendrik Degering and Denny Fliegner for computing support.

tobias.kreilos@physik.uni-marburg.de tobias.schneider@ds.mpg.de

[1] S. Grossmann, Rev. Mod. Phys. 72, 603 (2000).

[2] A. G. Darbyshire and T. Mullin, J. Fluid Mech. 289, 83 (1995).

[3] A. Schmiegel and B. Eckhardt, Phys. Rev. Lett. 79, 5250 (1997).

[4] B. Eckhardt, T. M. Schneider, B. Hof, and J. Westerweel, Annu. Rev. Fluid Mech. 39, 447 (2007).

[5] B. Eckhardt, Nonlinearity 21, T1 (2008).

[6] T. Kreilos and B. Eckhardt, Chaos 22, 047505 (2012).

[7] G. Kawahara, M. Uhlmann, and L. van Veen, Annu. Rev. Fluid Mech. 44, 203 (2012).

[8] C. Grebogi, E. Ott, and J. A. Yorke, Phys. Rev. Lett. 57, 1284 (1986).

[9] S. Bottin and H. Chaté, Eur. Phys. J. B 6, 143 (1998).

[10] B. Hof, J. Westerweel, T. M. Schneider, and B. Eckhardt, Nature (London) 443, 59 (2006).

[11] J. Peixinho and T. Mullin, Phys. Rev. Lett. 96, 094501 (2006).

[12] A. P. Willis and R. R. Kerswell, Phys. Rev. Lett. 98, 014501 (2007).

[13] B. Hof, A. de Lozar, D. J. Kuik, and J. Westerweel, Phys. Rev. Lett. 101, 214501 (2008).

[14] T. M. Schneider and B. Eckhardt, Phys. Rev. E 78, 046310 (2008).

[15] T. M. Schneider, F. De Lillo, J. Buehrle, B. Eckhardt, T. Dörnemann, K. Dörnemann, and B. Freisleben, Phys. Rev. E 81, 015301(R) (2010).

[16] R. R. Kerswell, Nonlinearity 18, R17 (2005).

[17] J. P. Crutchfield and K. Kaneko, Phys. Rev. Lett. 60, 2715 (1988). 
[18] L. P. Kadanoff and C. Tang, Proc. Natl. Acad. Sci. U.S.A. 81, 1276 (1984).

[19] Y. Duguet, a. P. Willis, and R. R. Kerswell, J. Fluid Mech. 613, 255 (2008).

[20] J. F. Gibson, J. Halcrow, and P. Cvitanović, J. Fluid Mech. 611, 107 (2008).

[21] J. Vollmer, T. M. Schneider, and B. Eckhardt, New J. Phys. 11, 013040 (2009).

[22] L. van Veen and G. Kawahara, Phys. Rev. Lett. 107, 114501 (2011).

[23] K. T. Allhoff and B. Eckhardt, Fluid Dyn. Res. 44, 031201 (2012).

[24] K. Avila, D. Moxey, A. de Lozar, M. Avila, D. Barkley, and B. Hof, Science 333, 192 (2011).

[25] F. Waleffe, Phys. Rev. Lett. 81, 4140 (1998).

[26] J. F. Gibson, Channelflow: A Apectral Navier-Stokes Simulator in $C++$ (University of New Hampshire, New Hampshire, 2012).

[27] M. Nagata, J. Fluid Mech. 217, 519 (1990).

[28] R. M. Clever and F. H. Busse, J. Fluid Mech. 344, 137 (1997).
[29] J. D. Skufca, J. A. Yorke, and B. Eckhardt, Phys. Rev. Lett. 96, 174101 (2006).

[30] T. M. Schneider, J. F. Gibson, M. Lagha, F. De Lillo, and B. Eckhardt, Phys. Rev. E 78, 037301 (2008).

[31] F. Mellibovsky and B. Eckhardt, J. Fluid Mech. 709, 149 (2012).

[32] M. Avila, F. Mellibovsky, N. Roland, and B. Hof, Phys. Rev. Lett. 110, 224502 (2013).

[33] A. Riols, F. Rincon, C. Cossu, G. Lesur, P.-Y. Longaretti, G. I. Ogilvie, and J. Herault, J. Fluid Mech. 731, 1 (2013).

[34] C. Grebogi, E. Ott, and J. A. Yorke, Physica (Amsterdam) 7, 181 (1983).

[35] Y.-C. Lai and C. Grebogi, Phys. Rev. E 49, 1094 (1994).

[36] Y.-C. Lai and T. Tél, Transient Chaos: Complex Dynamics on Finite Time Scales (Springer, New York, 2011).

[37] J. D. Hanson, J. R. Cary, and J. D. Meiss, J. Stat. Phys. 39, 327 (1985).

[38] G. Lemoult, L.-J. Aider, and J. E. Westfreid, Phys. Rev. E 85, 025303(R) (2012).

[39] Y. Tasaka, T. M. Schneider, and T. Mullin, Phys. Rev. Lett. 105, 174502 (2010). 